\title{
Reversibility of optic nerve damage in primate eyes subjected to intraocular pressure above systolic blood pressure
}

\author{
RONALD L. RADIUS AND DOUGLAS R. ANDERSON \\ From the Eye Institute, Medical College of Wisconsin, Milwaukee, Wisconsin; and the \\ William L. McKnight Vision Research Center, Bascom Palmer Eye Institute, \\ Department of Ophthalmology, University of Miami School of Medicine, Miami, Florida, USA
}

SUMMARY It was shown previously that pressure-induced interruption of axonal transport at the optic nerve head is reversible after 8 hours of raised intraocular pressure (perfusion pressure of 25 to $35 \mathrm{mmHg}$ ). Here we report experiments on the nature, location, and reversibility of pressureinduced abnormalities of the optic nerve head, including interruption in axonal transport, in eyes subjected to a more extreme pressure insult (perfusion pressure less than $25 \mathrm{mmHg}$ ), including some insults that produced total ischemia. After $1 / 2$ to 2 hours of intraocular pressure $10 \mathrm{mmHg}$ greater than ophthalmic artery systolic blood pressure there was hydropic swelling and tissue necrosis in the anterior lamina cribrosa, the optic nerve head, and the retina. Organelle accumulation, identifying the locus of interruption in retrograde fast axonal transport, occurred exclusively within the posterior one-third of the lamina cribrosa. The degree of these changes and the number of axons involved related to the duration of the raised pressure in eyes studied 24 hours after this severe pressure insult. These tissue changes appeared to have been irreversible after a 4-hour insult and partly irreversible after a 1- or 2-hour insult. In eyes studied 2 weeks later diffuse axon atrophy was apparent across the extent of the optic nerve section after a 4-hour insult, and there was partial atrophy after a 1- or 2-hour insult. After 4 hours of slightly lower intraocular pressure levels (perfusion pressure of 10 to $20 \mathrm{mmHg}$ ) the irreversible nerve head injury was less extensive; after 4 hours of even lower pressure levels (perfusion pressures of 25 to $30 \mathrm{mmHg}$ ) no permanent nerve injury was identified. Thus at least some axons can survive total ischaemia for 2 hours, which is longer than the ganglion cells of the retina can survive total ischaemia. A milder insult produces a partial injury that can be reversible after a much longer duration of the insult.

Several investigators have shown that raised intraocular pressure in primate eyes can interrupt optic nerve axonal transport in the optic nerve where it traverses the lamina cribrosa. ${ }^{1-8}$ This transport interruption may be the result of focal ischaemia as a consequence of a compromise of nerve head perfusion in these eyes with raised intraocular pressure. $^{278}$ Interestingly, even after 8 hours of raised intraocular pressure many experimental eyes show a reversal of these axonal transport abnormalities after return to normal of the intraocular pressure. ${ }^{17}$ For instance, in eyes examined 24 hours

Correspondence to Ronald L. Radius, MD. Medical College of Wisconsin, 8700 West Wisconsin Avenue, Milwaukee, Wisconsin 53226. USA. after the pressure insult (perfusion pressure $35 \mathrm{mmHg}$ for 6 to 8 hours) little if any residual accumulation of the material representing blocked transport remains at the lamina cribrosa. ${ }^{1}$ Similarly, in eyes studied one month after the pressure insult (perfusion pressure 25 $\mathrm{mmHg}$ for 4 hours) no axon atrophy, which would result from irreversibly blocked transport, is evident. ${ }^{7}$ On the other hand ischaemic insult to the retina and other neuronal tissues is typically irreversible after 2 hours of anoxia. -11

The reversibility of the injury at the optic nerve head could be explained by supposing that it is purely mechanical, without ischaemia. However, an alternative explanation might be that the transport abnormalities in these eyes with experimental glaucoma are 
secondary to neuronal ischaemia, but that axon segments at the nerve head might be more resistant to isehaemia than are ganglion cell bodies in the retina. Yet another explanation might be that the nerve head ischaemia at the pressure levels studied may be incomplete, sufficient to cause a focal interruption in axonal transport but inadequate to cause irreversible axon damage.

To investigate this question further we studied the optic nerve abnormalities that resulted when primate eyes were subjected to periods of extreme pressure that was certain to produce complete ischaemia of the nerve head and retina. We first looked at the morphology of the retina and optic nerve head after 1,2, and 4 hours of intraocular pressure above systolic blood pressure, recording the progressive accumulation of membranous bodies and organelles as a result of impaired axonal transport. We then examined a similar experimental group 24 hours after the 1,2 , or 4 hour interval of this severely raised pressure; in this group, we looked for the persistence or disappearance of the membranous organelles as a sign of nonreversibility or reversibility of the transport blockade produced by the pressure elevation. Finally, to determine the irreversible lethal effects of the pressure elevation we looked for axon necrosis 2 weeks after the 4-hour insult of severe pressure elevation. For comparison we examined the morphology of eyes 24 hours and 2 weeks after they had been subjected to 4 hours of pressure elevation that was less severe (perfusion pressure of 10 to $30 \mathrm{mmHg}$ ).

\section{Materials and methods}

In all, 44 owl monkey (Aotus trivirgatus) eyes were studied after various periods of raised intraocular pressure. The pressure levels were manipulated manometrically by adjusting the height of a fluid reservoir connected via polyethylene tubing to a 23gauge needle inserted into the anterior chamber. Ophthalmic artery pressure (systolic and diastolic) was determined by raising the fluid reservoir until the central retinal artery observed ophthalmoscopically pulsated (diastolic) and collapsed (systolic). Systemic blood pressure was monitored continuously by a pressure transducer connected to polyethylene tubing inserted into the right femoral artery. 100 units of sodium heparin was injected intra-arterially via this catheter. Mean femoral artery pressure was calculated as diastolic pressure plus one-third the difference between systolic and diastolic pressures. Anaesthesia of experimental animals was maintained throughout the study interval by an initial intramuscular injection of $0.1 \mathrm{ml}$ ketamine (Ketaset, $100 \mathrm{mg} / \mathrm{ml}$ ) and repeated intra-arterial injections of $0.1 \mathrm{ml}$ pentobarbital (Nembutal, $50 \mathrm{mg} / \mathrm{ml}$ ).
Eight eyes, 2 at each time interval, were subjected to $1 / 2,1,2$, and 4 hours of pressure elevation 10 $\mathrm{mmHg}$ above ophthalmic artery systolic pressure. During the interval the intraocular pressure was varied according to any changes in femoral artery pressure, on the assumption that the ophthalmic artery pressure changed in concert with the systemic blood pressure. At the end of the interval tissue was prepared for morphological examination.

An additional 11 eyes were similarly subjected to $1 / 2$ ( 2 eyes), 1 ( 5 eyes), 2 ( 2 eyes), and 4 ( 2 eyes) hours of severely increased intraocular pressure $(10 \mathrm{mmHg}$ greater than ophthalmic artery systolic pressure), followed by 24 hours of intraocular pressure reduced to atmospheric pressure before the tissue was prepared for examination. Reduction in intraocular pressure during this 24 -hour interval was maintained by a corneal fistula.

In a third experimental group each of 12 eyes was maintained at one of several ocular perfusion pressures (mean femoral artery pressure minus intraocular pressure) between 30 and $-10 \mathrm{mmHg}$. After 4 hours the intraocular pressure in these eyes was also reduced to atmospheric pressure by fistulisation of the anterior chamber. As in the second study group these experimental eyes were studied 24 hours after this pressure reduction.

Finally 13 additional eyes were subjected to 4 hours of raised intraocular pressure (perfusion pressures between 25 and $-5 \mathrm{mmHg}$ ), but studied 2 weeks later. After the 4 hours of high pressure the intraocular pressure in these eyes was restored to normal manometrically, and the needles were removed. Subsequently the intraocular pressure was measured on the first, third, fifth, seventh, tenth, and fourteenth day after the pressure insult, and on no occasion were any of these eyes found to have a pressure greater than $25 \mathrm{mmHg}$. In selected eyes fluorescein angiography was performed before, during, and after the interval of pressure elevation.

In all study groups histological material was fixed in situ by perfusion with $200 \mathrm{ml}$ of normal saline followed by $300 \mathrm{ml}$ of $4 \%$ buffered glutaraldehyde fixative ( $\mathrm{pH} \mathrm{7.4)} \mathrm{retrograde} \mathrm{via} \mathrm{the} \mathrm{abdominal} \mathrm{aorta}$ into the carotid arteries. This perfusion was carried out at a hydrostatic pressure of $180 \mathrm{mmHg}$ with the intraocular pressure at $10 \mathrm{mmHg}$. A right ventricular cardiac incision allowed egress of blood, saline, and fixative during the tissue perfusion. All experimental eyes were enucleated immediately, and tissue blocks containing the optic nerve head were excised. These specimens were postfixed in osmium tetroxide and embedded in epoxy resin (Epon) for routine sectioning. Thick sections $(2 \mu \mathrm{m})$ containing the optic nerve head were stained with paraphenylene diamine and examined by phase contrast microscopy. Thin 
sections, chosen from those regions of the lamina cribrosa that appeared to have the most tissue changes following the pressure elevations, were counterstained with uranyl acetate and lead citrate for electron microscopic examination. Qualitative scores from 0 to $4+$ were assigned to these specimens according to the amount of organelle accumulation (mitochondria, microvesicles, dense bodies) seen at the level of the lamina cribrosa. This material aggregation was interpreted to reflect the presence and degree of impaired axonal transport mechanisms in these hypertensive eyes. ${ }^{16}$

\section{Results}

Observations after $1 / 2$ to 4 hours of pressure elevation In all eyes studied at intraocular pressures $10 \mathrm{mmHg}$ above ophthalmic artery systolic pressure the ocular blood flow to the choroid, retina, and optic nerve head had been eliminated. Fluorescein angiograms performed while the intraocular pressure was raised

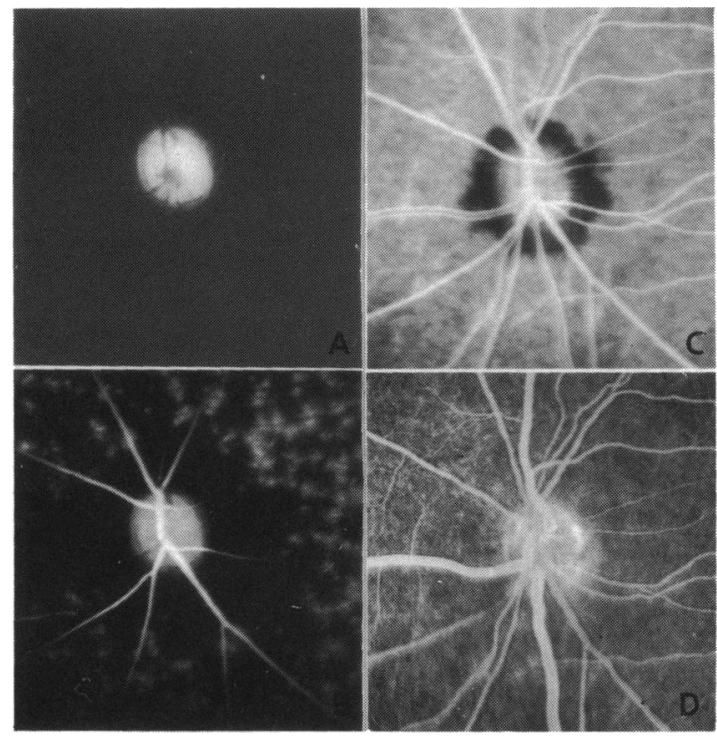

Fig. 1 Fluorescein angiograms of primate eyes with manometrically raised intraocular pressure. In eyes with pressure elevation of $10 \mathrm{mmHg}$ greater than ophthalmic artery systolic pressure no fluorescein enters either the retinal or choroidal circulation (A). Fluorescence is, however, noted at the nerve head in these eyes. Whether the pressure is lowered in a step-wise fashion, $2 \mathrm{mmHg}$ at a time, or precipitously, the central retinal artery and choroidal vasculature fill almost simultaneously (B). The peripapillary choroidal vasculature has a persistent defect even when there is retinal arteriovenous filling $(\mathrm{C})$; however, this hypofluorescent filling pattern is no longer seen in the late venous angiogram sequence (D). showed no movement of dye into the experimental eye (Fig. 1A). As the intraocular pressure was lowered the central retinal artery and peripheral choroidal vasculature filled with fluorescein essentially simultaneously (Fig. 1B). Filling of the nerve head vasculature occurred at an intraocular pressure identical to (or at most 3 to $5 \mathrm{mmHg}$ less than) that at which the central retinal artery perfusion had been restored. It took considerable reduction of intraocular pressure to allow filling of the peripapillary choroidal vasculature (Fig. 1C and D). Even if the pressure was reduced precipitously from maximal levels to zero, delay in filling of this vascular bed was seen.

By phase-contrast microscopy retinal specimens from eyes subjected to $1 / 2$ hour of pressure elevation had mild cytoplasmic vacuolisation that was progressively worse and associated with nuclear changes after longer periods of ischaemia (Fig. 2). The changes in the inner retina, as expected, followed a course similar to that observed after central retinal artery occlusion. ${ }^{9}$

At the optic nerve head after $1 / 2$ hour of pressure some of the optic nerve axons and mitochondria were swollen (Fig. 3). Glial elements had less severe morphological alterations. These changes were more severe after longer durations of ischaemia. This hydropic degeneration of neural elements extended to the posterior half of the lamina cribrosa.

Within the region of the posterior third of the lamina cribrosa and the most anterior portion of the myelinated optic nerve additional changes were seen that represent blocked retrograde axonal transport. After only $1 / 2$ hour of pressure elevation mitochondria, dense bodies, and membranous material had accumulated within isolated bundles of axons in this region (Fig. 4). The amount of accumulated material increased with time and was associated with loss of cellular integrity.

\section{Reversibility of changes produced by severe pressure elevation}

Eleven additional eyes were subjected to pressure elevation above systolic blood pressure followed by 24 hours of reduced intraocular pressure before microscopic examination. In order to determine whether the restoration of normal intraocular pressure allowed ocular reperfusion in these eyes, fluorescein angiography was performed immediately after the intraocular pressure was reduced to 10 $\mathrm{mmHg}$. In those specimens which had only $1 / 2$ or 1 hour of pressure elevation angiograms showed prompt reperfusion of the retina, optic nerve head, and choroid.

Frequently there was some leakage of dye at or near the disc margin and from the major retinal 
Fig. 2 Photomicrographs of retina of eyes subjected to intraocular pressure $10 \mathrm{mmHg}$ greater than ophthalmic artery pressure for $1 / 2$ hour (A), 2 hours (B), and 4 hours $(\mathrm{C})$. After $1 \frac{1}{2}$ hour of high pressure elevation cytoplasmic vacuolisation can be seen mainly in the retinal nerve fibre layer (NFL), the ganglion cell layer (GCL), and the inner nuclear layer (INL). After 2 hours of pressure insult these cytoplasmic changes are even more extensive, and scattered nuclei are pyknotic. After 4 hours of pressure elevation general disruption of all retinal layers is evident. This includes hydropic swelling of cell bodies within all 3 retinal nuclear layers and loss of cellular detail within the retinal nerve fibre layer and the photoreceptor layer $(P R L)$. In addition, pyknotic and granular degeneration of nuclei is noted, especially within the ganglion cell and inner nuclear layer. (Paraphenyldiamine, $\times 1000$ ).

vasculature (Fig. 5), as noted in previous experiments. ${ }^{7}$ Unfortunately in eyes subjected to 2 or 4 hours of high pressure corneal clouding and anterior chamber reaction usually prevented obtaining an adequate fluorescein angiogram. In those specimens which could be successfully studied there was normal filling of the choroidal vasculature, but a detectable delay in filling of the retinal and nerve head vessels was noted. Later frames of the angiogram sequence showed filling of the retinal vasculature bed, but resolution in these frames was inadequate to study the adequacy of nerve head perfusion.

After the interval of high pressure, the angiographic study, and 24 hours of reduced pressure the findings in the retina and prelaminar part of the optic nerve head were as follows: In eyes that had been subjected to $1 / 2$ hour of pressure elevation, isolated axons within the retina and at the nerve head were mildly swollen with dissolution of cytoplasmic detail. In many fibres swollen mitochondria were noted (Fig. 6). In those eyes studied 24 hours after 1 or 2 hours of pressure elevation more axons had swollen mitochondria and loss of cytoplasmic details (Fig. 6), some with gross distortion and rupture of the axonal membrane. However, within a single specimen regions with marked axon changes were intermixed with areas with little evident damage. After 4 hours of high pressure followed by 24 hours of low intraocular pressure severe hydropic degeneration and loss of cellular outline throughout the retina and nerve head was seen. In general, glial elements were less profoundly affected in all eyes at each of the several time periods studied.

More posteriorly, within the region of the lamina cribrosa, the additional tissue changes of blocked axonal transport were seen. In specimens studied 24 hours after $1 / 2$ hour of pressure elevation the axons within the region of the lamina scleralis looked essentially normal, showing that the micro-organelles
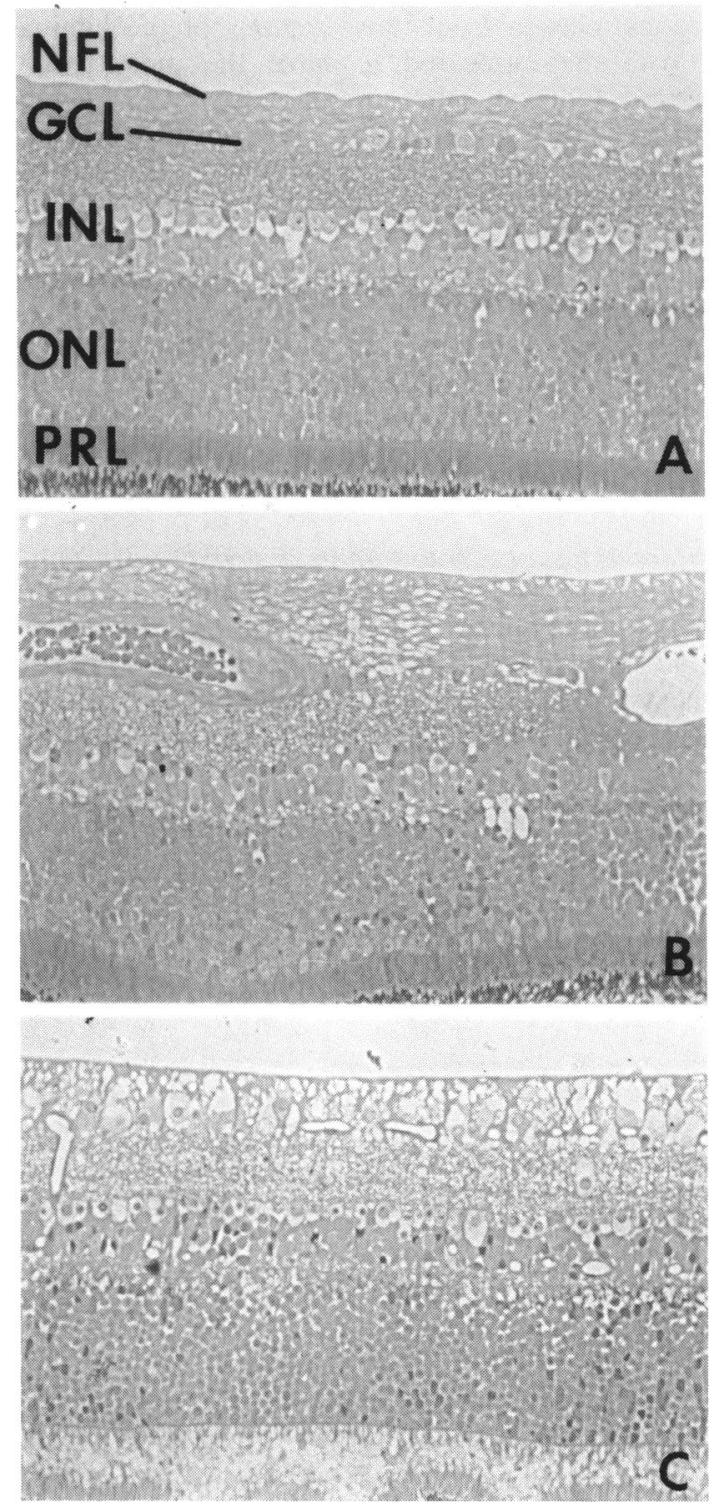

known from the previous experimental group to accumulate during $1 / 2$ hour of pressure elevation had disappeared. However, in specimens subjected to longer periods of pressure elevation (1 to 2 hours), accumulation of mitochondria, microvesicles, and dense membranous material persisted despite 24 hours of low pressure (Fig. 7). The degree of accumulation was equal to or slightly greater than that seen in specimens studied immediately after comparable high pressure intervals (Tables 1 and 2). After 4 hours of high pressure followed by 24 hours of low pressure, accumulation of organelles at the lamina was even 

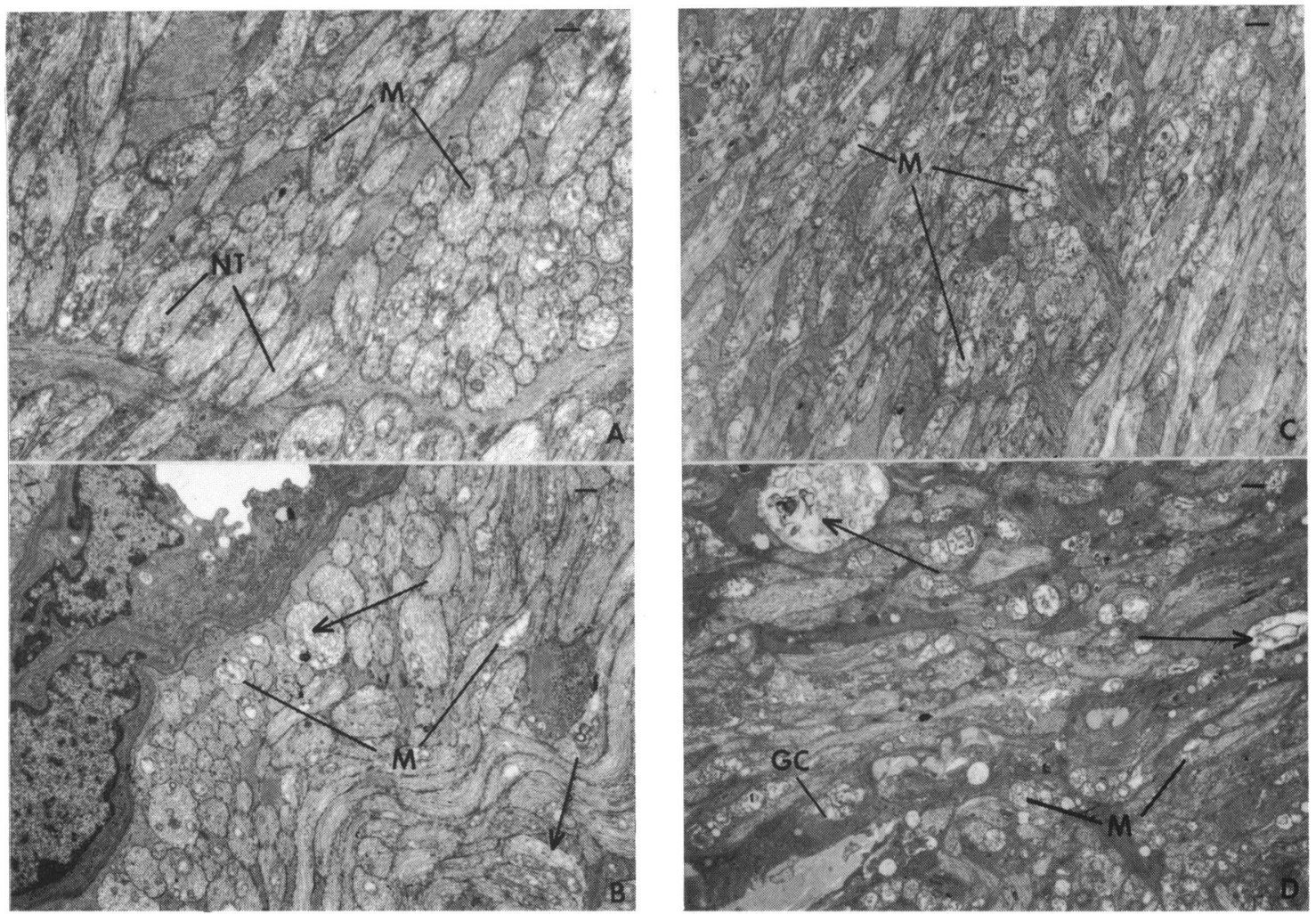

Fig. 3 Electron photomicrographs of specimens selected from the region of the optic nerve head anterior to the lamina cribrosa. In sections from normal eyes (A) the axons at the optic nerve head contain on the average 2 or 3 mitochondria (M), together with a cytoplasmic matrix of neurotubular (NT) elements. After $1 / 2$ hour of maximal pressure elevation $(10 \mathrm{mmHg}$ greater than ophthalmic artery systolic pressure) early degenerative changes are apparent, such as swelling of axon mitochondria $(M)$, and some disorganisation of the normal neurotubular cytoarchitecture (arrows, B). These changes are even more pronounced after 2 hours of high-pressure insult (C). After 4 hours of maximal pressure elevation (D) degenerative changes within this region of the nerve head involve nearly all axons, as well as occasional glial cells (GC). In some instances gross swelling of axons with disruption of cytoplasmic membranes and loss of all cellular integrity is seen (arrows). ( $\times 2750)$.

more striking, involving the entire nerve head (Fig. 7). Although accumulation of material within axons was predominantly within the region of the lamina cribrosa, occasionally axons within the retinal nerve fibre layer had similar changes (Fig. 8).

In eyes examined immediately after pressure insult, as well as those studied after 24 hours of ocular hypotony, patent microvasculature was evident within regions of axonal degeneration and organelle accumulation (Fig. 7). There was no evidence of endothelial cell swelling or capillary closure in any of the specimens examined after any of the 4 intervals $(1 / 2,1$, 2 , and 4 hours).

Reversibility of nerve head injury from less severe pressure elevation for 4 hours

Twelve eyes were subjected to 4 hours of elevation of intraocular pressure that was less severe (perfusion pressure between 30 and $-10 \mathrm{mmHg}$, Table 3). After 4 hours this intraocular pressure was reduced to that of atmospheric pressure for $\mathbf{2 4}$ hours by means of a surgical fistula. In most eyes studied at the higher perfusion pressures (lower intraocular pressures) there was little or no accumulation of microorganelles when specimens were examined 24 hours after the pressure insult. Scattered inflammatory cells and extravasated red blood cells could be identified within the retina and anterior optic nerve head, but no other abnormalities were seen. On the other hand eyes studied 24 hours after 4 hours at a perfusion pressure of 15 to $10 \mathrm{mmHg}$ had hydropic swelling of nerve head tissue elements, especially immediately anterior to the lamina cribrosa, as well as trace to $2+$ accumulation of micro-organelles within the region of 

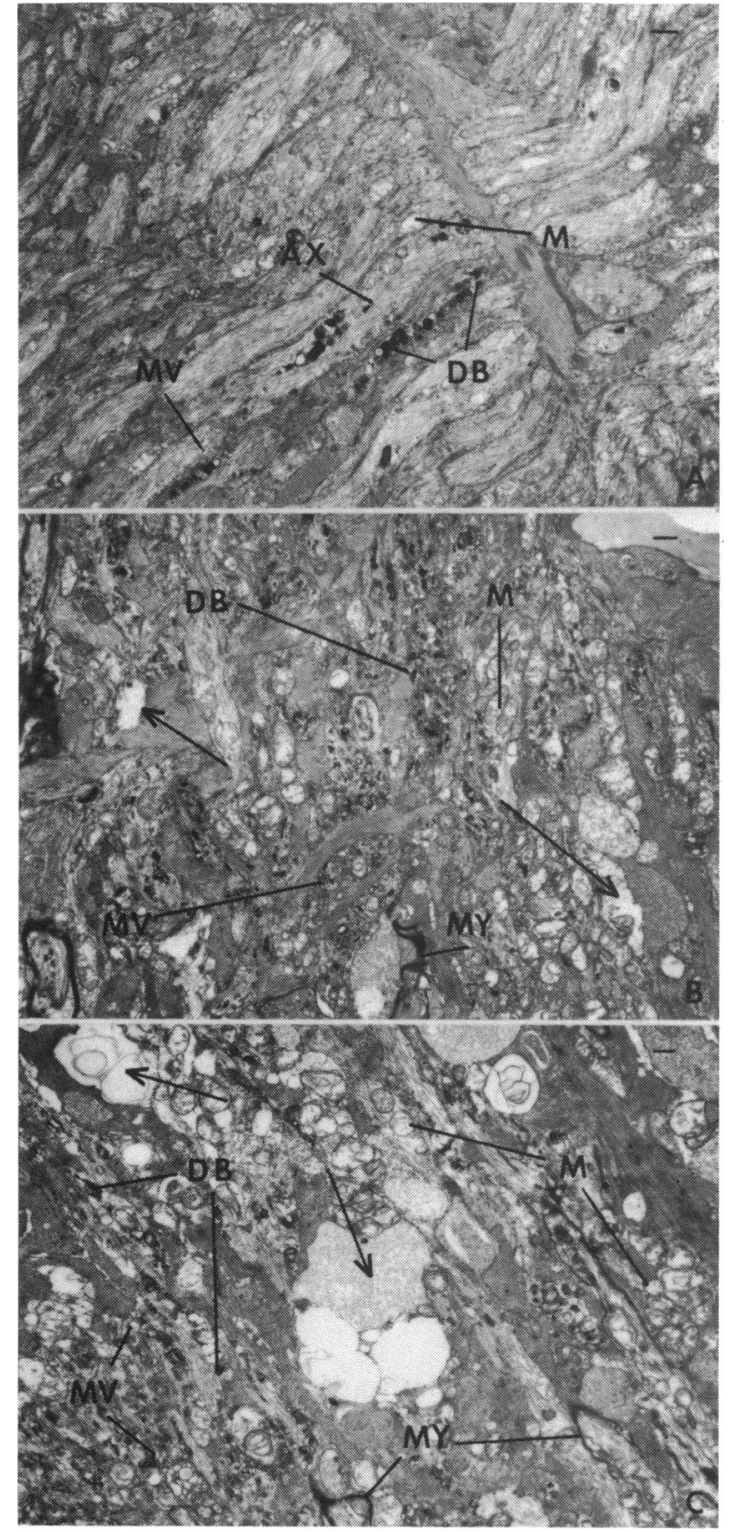

the lamina itself. These changes were similar to those seen 24 hours after 4 hours of severe pressure elevation (Fig. 7).

At the lowest perfusion pressure ( 5 to $-10 \mathrm{mmHg}$ ) hydropic swelling of axon segments was more widespread in the anterior optic nerve head, together with rupture of mitochondrial and cytoplasmic membranes and general dissolution of cellular detail. In these same eyes, there were $3+$ to $4+$ accumulation of micro-organelles at and immediately posterior to
Fig. 4 Electron photomicrographs from the posterior half of the lamina cribrosa of eyes subjected to intraocular pressures $10 \mathrm{mmHg}$ greater than ophthalmic artery systolic pressure. After $1 / 2$ hour of pressure insult (A), scattered axons $(A x)$ are seen to contain an accumulation of dense bodies. $(D B)$, microvesicles $(M V)$, and mitochondria $(M)$. After 2 hours of pressure elevation (B), extensive accumulation of microorganelles is present, involving essentially all portions of the lamina cribrosa. Furthermore, axons show significant degenerative changes, such as fibre and mitochondria swelling with membrane disruption and dissolution of cytoplasmic detail (arrows). After 4 hours of pressure elevation (C) all fibre bundles within the region are more severely damaged (My, myelin). (× 2750).

Table 1 Organelle accumulation in primate eyes subjected to periods of high intraocular pressure $(10 \mathrm{~mm} \mathrm{Hg}$ greater than ophthalmic artery systolic pressure)

\begin{tabular}{lll}
\hline $\begin{array}{l}\text { Duration of } \\
\text { pressure elevation }\end{array}$ & Eve & $\begin{array}{l}\text { Degree of } \\
\text { organelle accumulation }\end{array}$ \\
\hline $1 / 2$ hour & 617 OS & $1+$ \\
1 hour & 531 OD & Trace \\
& 562 OD & $1+$ \\
2 hours & 610 OD & Trace \\
& 568 OD & $2+$ \\
4 hours & 403 OD & $1+$ \\
& 525 OD & $4+$ \\
\hline
\end{tabular}

Table 2 Organelle accumulation in primate eyes subjected to high pressure followed by 24 hours of ocular hypotony (10 $\mathrm{mm} \mathrm{Hg}$ greater than ophthalmic artery systolic pressure)

\begin{tabular}{lll}
\hline $\begin{array}{l}\text { Duration of } \\
\text { pressure elevation }\end{array}$ & Eve & $\begin{array}{l}\text { Degree of persistent } \\
\text { organelle accumulation }\end{array}$ \\
\hline $1 / 2$ hour & 529 OD & Trace \\
& 531 OS & Trace \\
1 hour & 403 OS & 0 \\
& 511 OD & $1+$ \\
& 529 OS & 0 \\
& 547 OS & $1+$ \\
2 hours & 562 OS & $2+$ \\
& 513 OD & $1+$ \\
4 hours & 546 OS & $4+$ \\
& 525 OS & $3+$ \\
\hline
\end{tabular}

the lamina cribrosa. In some specimens cellular necrosis extended posterior to the beginning of the retrolaminar optic nerve. Outer retinal elements and choroidal tissues were significantly damaged, especially at the higher levels of intraocular pressure, but the changes were never more severe than the damage to the inner retina and the optic nerve head.

Atrophy following irreversible injury

Finally 13 eyes were subjected to raised intraocular 


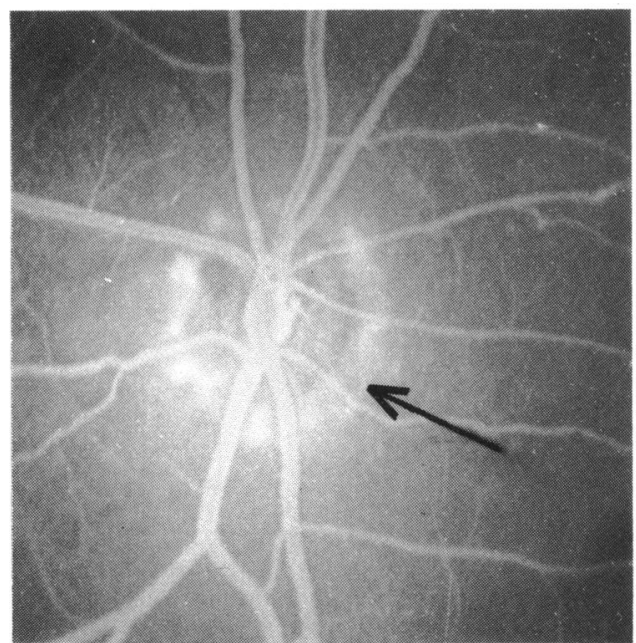

Fig. 5 Fluorescein angiogram performed immediately after 1 hour of intraocular pressure $10 \mathrm{mmHg}$ greater than ophthalmic artery systolic pressure. The intraocular pressure was reduced to $10 \mathrm{mmHg}$ just prior to injection of the fluorescein. Though the clarity of the media is somewhat decreased after this period of high-pressure elevation, reperfusion of choroidal, retinal, and nerve head vasculature is apparent in this arteriovenous phase angiogram. Some leakage from peripapillary vasculature is present (arrow).

Fig. 6 Electron photomicrographs of optic nerve head from monkey eyes subjected to high intraocular pressure followed by 24 hours of reduced pressure. Tissue specimens are taken from the region of the optic nerve head immediately anterior to the scleral lamina cribrosa. In eyes that had been subjected to a $1 / 2$ hour interval of pressure elevation minimal nerve head changes are evident after 24 hours of reduced intraocular pressure (A). Swollen mitochondria (M) are seen in several axons $(A x)$ in addition to swelling and dissolution of cytoplasmic ultrastructure in selected nerve fibre (arrows). Eyes that had suffered 2 hours of pressure elevation have more extensive nerve head changes when examined 24 hours later (B). In such instances most axons have some evidence of injury including swelling of mitochondria $(M)$ and disruption of neurotubular structure (arrows). In addition many axons are markedly swollen, with rupture of cytoplasmic membranes and loss of cellular integrity. In eyes that had been exposed to 4 hours of pressure elevation this hydropic degeneration is even more extensive (C). In many areas of the nerve head loss of all recognisable axon structure is present. Swelling of cytoplasmic organelles is seen in glial $(G C)$ elements, as well, after this degree of pressure insult. $(\times 2750)$. pressure at one of several perfusion pressures as in the previous sections. after 4 hours of high pressure the intraocular pressure was reduced to $10 \mathrm{mmHg}$ manometrically, the infusion needle was removed from the eye, and the tissue was examined 2 weeks later (Table 4). The intraocular pressure never exceeded 25 $\mathrm{mmHg}$ during the next 2 weeks. With one exception the eyes studied at a perfusion pressure of $15 \mathrm{mmHg}$ or higher had little or no evidence of retinal or optic nerve atrophy (Table 4). In the 3 eyes studied at a perfusion pressure of $10 \mathrm{mmHg}$ there was diffuse

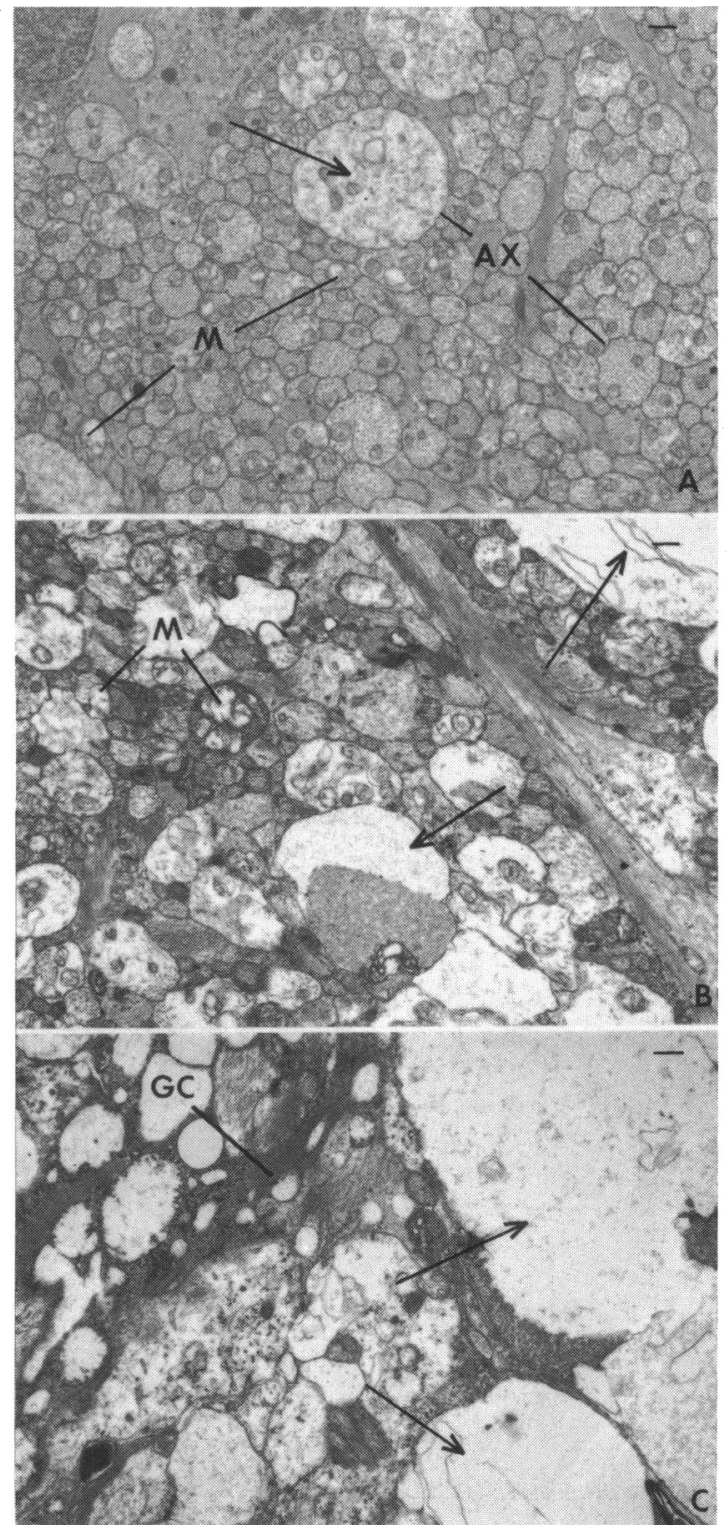


optic nerve atrophy with damaged fibres intermixed with normal axons throughout the nerve cross-section (Fig. 9). No particular region of the nerve seemed to have been particularly susceptible to the pressure elevation. This pattern is quite similar to what might be expected if eyes with clinical transient high pressure elevation could be studied histologically. ${ }^{12}$ This distribution of axon damage is in contrast, however, to that observed in experimental as well as clinical instances of pressure elevation of a more moderate degree in which nerve abnormalities appear
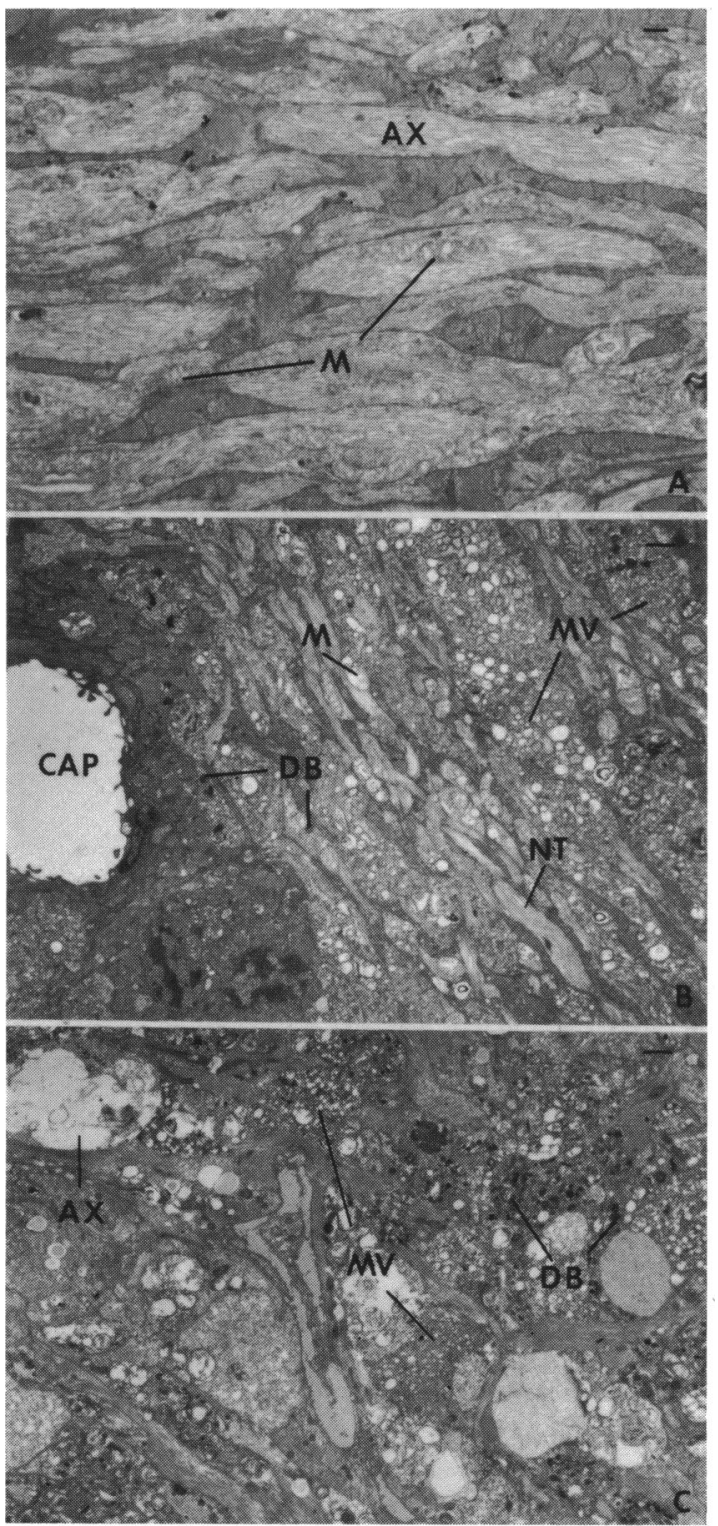

Table 3 Retinal and nerve head tissue damage including persistent organelle accumulation in primate eyes studied 24 hours after a 4-hour interval of high intraocular pressure

\begin{tabular}{lllll}
\hline $\begin{array}{l}\text { Perfusion } \\
\text { pressure }\end{array}$ & Eye & $\begin{array}{l}\text { Degree of } \\
\text { organelle } \\
\text { accumulation }\end{array}$ & \multicolumn{2}{l}{ Tissue changes } \\
\cline { 5 - 6 } & & Retina & Optic nerve \\
\hline $30 \mathrm{mmHg}$ & 211 OD & 0 & 0 & 0 \\
$25 \mathrm{mmHg}$ & 575 OS & $2+$ & 0 & $2+$ \\
$15 \mathrm{mmHg}$ & 583 OS & $1+$ & 0 & $2+$ \\
& 587 OS & $2+$ & 0 & $1+$ \\
$10 \mathrm{mmHg}$ & 588 OD & Trace & 0 & $1+$ \\
& 585 OS & $2+$ & 0 & $1+$ \\
& 582 OD & Trace & 0 & $1+$ \\
$5 \mathrm{mmHg}$ & 582 OS & Trace & $1+$ & $1+$ \\
& 574 OD & $3+$ & $2+$ & $3+$ \\
$0 \mathrm{mmHg}$ & 581 & $4+$ & $1+$ & $3+$ \\
$-10 \mathrm{mmHg}$ & 525 OS & $4+$ & $4+$ & $4+$ \\
& 530 OS & $3+$ & $4+$ & $4+$ \\
\hline
\end{tabular}

Table 4 Retinal and optic nerve atrophy in primate eyes subjected to 4 hours of high-pressure elevation followed by 2 weeks at reduced pressure

\begin{tabular}{llll}
\hline Perfusion pressure & Eye & \multicolumn{2}{l}{ Tissue atrophy } \\
\cline { 3 - 4 } & & Retina & Optic nerve \\
\hline $25 \mathrm{mmHg}$ & & 0 & 0 \\
$20 \mathrm{mmHg}$ & 461 OD & 0 & 0 \\
$15 \mathrm{mmHg}$ & 598 OD & $4+$ & $4+$ \\
& 221 OD & 0 & 0 \\
$10 \mathrm{mmHg}$ & 597 OD & 0 & $1+$ \\
& 589 OS & 0 & $2+$ \\
$5 \mathrm{mmHg}$ & 587 OS & 0 & $1+$ \\
& 588 OS & $4+$ & $4+$ \\
& 600 OD & 0 & $3+$ \\
& 205 OD & 0 & $1+$ \\
$0 \mathrm{mmHg}$ & 598 OS & 0 & $3+$ \\
$-10 \mathrm{mmHg}$ & 200 OD & $2+$ & $1+$ \\
& 589 OD & $4+$ & $4+$ \\
\hline
\end{tabular}

Fig. 7 Electron photomicrographs from the region of the scleral lamina cribrosa examined after an intraocular pressure $10 \mathrm{mmHg}$ greater than ophthalmic artery systolic pressure, followed by 24 hours of reduced pressure. In eyes subjected to $1 / 2$ hour of such pressure insult (A) very few axon $(A x)$ changes are evident 24 hours later. There is no significant fibre swelling or disruption of normal cytoarchitecture, at least as seen by routine electron microscopy. There is questionable mitochondrial (M) swelling and minimal accumulation of micro-organelles, including dense bodies and smooth vesicle material. In eyes examined after 2 hours of pressure elevation (B), however, many axons have persistent accumulation of swollen mitochondria (M), microvesicles $(M V)$, and dense bodies (DB). Some axonal swelling is present but neurotubular (NT) elements are generally well preserved. Patent capillaries (cap) and normal endothelial cell morphology suggest that tissue reperfusion had been present during the 24-hour period of reduced pressure. In those eyes subjected to 4 hours of pressure elevation (C) extensive degenerative changes, as well as persistent accumulation of transport material, are seen even after 24 hours of ocular hypotony. There is gross swelling of many axonal (Ax) elements with membrane rupture and general loss of cellular integrity. Accumulation of micro-organelles including microvesicles $(M V)$, dense bodies $(D B)$, and swollen mitochondria are seen throughout the nerve head section. $(\times 2750)$. 


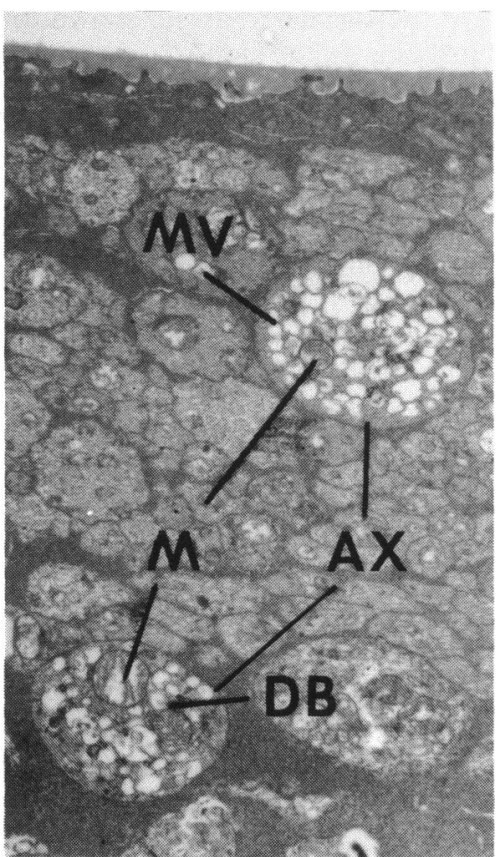

Fig. 8 Electron photomicrograph from the retinal nerve fibre layer 24 hours after a 1-hour interval of intraocular pressure $10 \mathrm{mmHg}$ greater than ophthalmic artery systolic pressure. Although most of the axons are normal, this retinal specimen shows 2 swollen axons $(A x)$ containing micro-organelle accumulation, including mitochondria $(M)$, microvesicles $(M V)$, and dense bodies $(D B)$, suggesting a local persistent abnormality of axonal transport mechanisms. $(\times 5000)$.

Fig. 9 Cross-sections of the myelinated portion of the optic nerve of primate eyes that 2 weeks earlier had been subjected to pressure insults (perfusion pressure between 25 and -10 $\mathrm{mm} \mathrm{Hg}$ ) for 4 hours. In eyes studied after moderate pressure elevation (perfusion pressure $20 \mathrm{mmHg}$ ) only rare degenerated axons (arrows) are noted (A). In specimens from eyes subjected to higher pressure levels (perfusion pressure $5 \mathrm{mmHg}$ ) a larger proportion of axons are atrophic throughout the optic nerve cross-section (arrows, B). Normal and damaged fibres are intermixed throughout the nerve head. There was no increased susceptibility to the pressure insult in any particular region of the nerve cross-section. In eyes studied at even more extreme pressure levels (perfusion pressure $-10 \mathrm{mmHg}$ ), essentially total optic atrophy is present $(\mathrm{C})$ with normal-appearing axon cylinders (arrows) rarely noted. (Paraphenylene diamine, $\times 110)$. to predominate in the superior and inferior temporal quadrants of the nerve head. ${ }^{13}{ }^{14}$ Some of the eyes studied at the lowest perfusion pressures (between 5 and $-10 \mathrm{mmHg}$ ) had only moderate tissue atrophy, but many eyes had nearly total optic atrophy with loss of axons in the retina and at the optic nerve head (Fig. 9 ). In one specimen the retinal changes seemed to exceed those of the optic nerve cross-section. However, in 6 of 13 eyes the nerve head seemed more damaged than the retina, and in 6 specimens the changes in the retina and optic nerve seemed equal.

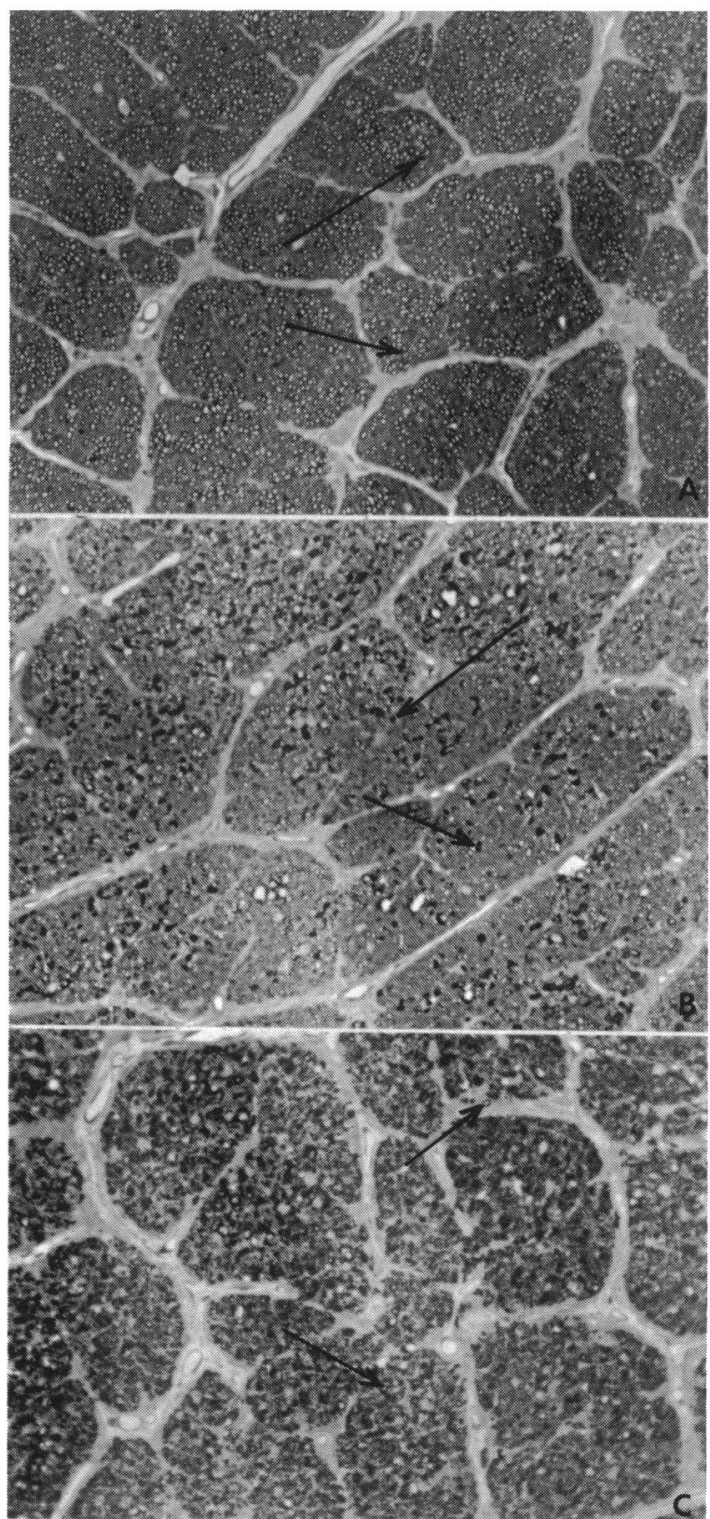




\section{Discussion}

Severe increase in intraocular pressure (intraocular pressure $10 \mathrm{mmHg}$ greater than ophthalmic artery systolic pressure) produced progressive retinal, nerve head, and optic nerve damage. There was hydropic degeneration of cellular elements with dilatation of axon cylinders and mitochondria, as well as general disorganisation and even disruption of normal cytoarchitecture. These changes are similar to tissue damage noted in eyes studied after $3 \frac{1}{2}$ hours of ocular ischaemia following occlusion of the central retinal artery and to the ischaemic changes seen in many tissues. ${ }^{9}$ We therefore believe them to be the consequence of tissue anoxia in the presence of the severely raised pressure.

In addition to the tissue changes there was a progressive accumulation of micro-organelles within the posterior third of the lamina cribrosa in these eyes. Such an accumulation of mitochondria, dense bodies, and microvesicles has been noted previously in eyes studied after periods of moderate pressure elevation and identifies axons with pressure-induced interruption of orthograde and retrograde axonal transport mechanisms. ${ }^{1-461516}$ It has so far been impossible to determine if this transport interruption with submaximal pressure elevation is a result of tissue anoxia or if it is due to more direct mechanical effects of the raised pressure.

In the present experiments the presence of ischaemia is certain, assured by the experimental conditions, and specifically confirmed by fluorescein angiography. Since the ganglion cells and retinal nerve fibre layers are ischaemic, orthograde transport mechanisms cannot make a contribution to the organelle accumulation at the lamina. Therefore, in the present experiments the accumulated material must represent cellular constituents carried there by the retrograde axonal transport mechanisms. Morphologically this material is not noticeably different from that seen in eyes with interruption in fast orthograde transport mechanisms, ${ }^{16}$ except perhaps a debatable preponderance of dense bodies with a reduction of microvesicular material.

This material accumulated at the most posterior region of the lamina cribrosa scleralis, signifying that the raised intraocular pressure can affect blood flow that far posteriorly and no further. Similar conclusions have been drawn from tissue pressure measurements in experimental eyes. ${ }^{17}$ The total blood flow in the lamina cribrosa (measured with microspheres) was not affected by severely raised intraocular pressure, ${ }^{18}$ presumably because the decreased flow in the innermost layers was balanced by a reactive increase in flow in the outer layers. The latter represents a zone of hyperaemia at the boundary of the ischaemic tissue, shown to exist in the nerve just outside the globe. ${ }^{18}$

It would seem that the blockage of axonal transport at the lamina cribrosa is reversible after half an hour of total ischaemia (compare Tables 1 and 2): the material that accumulated during the half-hour had dissipated 24 hours later (or at least did not continue to accumulate). Similarly, after a 1-hour or 2-hour insult the blockage did not persist (since additional material did not accumulate over the next 24 hours). However, especially after the 2-hour insult the accumulated material failed to dissipate completely. Thus there was an irreversible component to the injury. Conceivably some axons recovered and cleared up their accumulations, while others continued to accumulate material to an equivalent degree. Alternatively, although fluorescein angiography showed reperfusion of the nerve when the pressure returned to normal, there may have been scattered areas of nonreperfusion, so that some axons had a persisting (and therefore irreversible) injury represented by scattered areas of persistent intra-axonal accumulations.

The critical observation is not that there is some irreversible injury but that some, or even most, of the axons did recover after 2 hours of assured ischaemia, which means that axons can resist ischaemia longer than nerve cell bodies. The retinal ganglion cells, for example, cannot withstand 2 hours of ischaemia after central retinal artery occlusion. ${ }^{910}$

After 4 hours of ischaemia in the present experiment there was a maximal accumulation of material (Table 1), and the accumulation was not discernibly less 24 hours later (Table 2). We take this to mean that the axon injury after a 4-hour insult of total ischaemia is irreversible, and naturally 2 weeks later the optic nerve was completely atrophic (Table 4). This might be due to an irreversible axon injury, an irreversible injury to the ganglion body, or both. Unfortunately we cannot be certain that after 4 hours of high pressure there would be successful restoration of blood flow on lowering the intraocular pressure, because corneal oedema prevented our obtaining a technically adequate angiogram. Thus, if there was nonreperfusion or a delay in reperfusion, the insult that proved lethal might have been longer than 4 hours in duration; we can say, though, that at least by 24 hours there was no histological evidence of persistent capillary closure, occlusion, or even endothelial cell injury.

In any event it is clear that in this experimental situation axonal transport mechanisms fail to recover if the intraocular pressure is greater than systolic blood pressure (perfusion pressure of $-10 \mathrm{mmHg}$ or less) for 4 hours. However, axonal transport abnormalities are reversible if the 4-hour insult (or even a 6- 
to 8-hour insult) is less severe, that is, with a perfusion pressure of $25 \mathrm{mmHg}$ or more. This is shown by previous experiments ${ }^{17}$ dealing with rapid orthograde transport, and by the present data (Table 3 ). In the intermediate range (perfusion pressure of zero to $25 \mathrm{mmHg}$ ) the pressures are sufficient to have regularly caused transport blockade. ${ }^{278}$ In some of these eyes (Table 3) a partial block persists for 24 hours, though some of the injury was obviously reversible, since the degree of accumulation is less than would have been expected after a 4-hour insult based on our previous experience.

Of interest is that the findings at 24 hours (Table 3 ) show persistent axon swelling in the nerve head but not in the retina. This means that a threshold 4-hour insult can produce irreversible damage to the disc without permanent damage to the retina, even though the pressure-level sensitivities of the retina and optic nerve to a prolonged 8-hour pressure insult are similar $^{19}$ and even though the nerve head axons can withstand (at least partly) total ischaemia for 2 hours, which the retina cannot. Additional evidence that the nerve head is selectively sensitive under the present experimental conditions is the fact that the optic nerve showed evidence of mild to moderate atrophy at 2 weeks without equivalent changes in the retina (Table 4), with 2 exceptions that showed total atrophy. The 2-weeks interval would have allowed atrophy ascending from the point of injury to occur, but descending atrophy into the retina from the point of injury would not yet be evident. ${ }^{20-22}$

Overall, then, the irreversibility of the blockade of axonal transport seems to be related both to the severity and to the duration of the insult. Partial irreversibility may occur with either a severe insult of short duration or a moderate insult of longer (4 hours) duration. In the case of the maximal insult the fact that some axons recover indicates that total ischaemia for 2 hours is not a universally lethal insult to axons. The fact that some do succumb after 2 hours suggests either that some axons are damaged by a 2-hour ischaemic insult or that the insult was prolonged in certain zones because of scattered loci of sustained nonperfusion.

In the case of the 4-hour insult of sub-maximal severity partial irreversibility may indicate, for example, that in scattered loci there had been nearly total ischaemia for the 4 hours, while in other places there was partial ischaemia of insufficient degree and duration to result in a nonreversible injury. Alternatively, in those animals with a submaximal insult there may not be any pressure-induced ischaemia, but the transport is impaired by a mechanical compression or kinking of the axons. This type of insult might be reversible after 4 hours, whereas an ischaemic insult would not have been.
In summary, we had hoped to show that after an insult of total ischaemia for 4 hours the injury to axons would be completely reversible, even though the injury to the retinal cell body would be permanent. This would have offered an explanation for how pressure-induced axonal transport blockade could be reversible, namely, that an appropriate pressure would have produced a reversible ischaemic insult at the disc without a simultaneous ischaemia of the retina. As it happens the results were not so definitive. The data do show that some axons can survive 2 hours of total ischaemia, establishing that indeed they can withstand a more prolonged total ischaemia than retinal ganglion cells. However, it appears that the axons cannot survive 4 hours of total ischaemia. Does this mean that pressure-induced blockage of axonal transport that is reversible after 6 hours is not the result of ischaemia but of a pressureinduced mechanical effect? Certainly that is one possible explanation, but also possible is that the irreversible effect after 4 hours of high pressure in the present study is due to nonreperfusion. In that case reversibility with a less severe insult may signify that then there can be prompt reperfusion. A third alternative is that the ischaemia produced by a submaximal pressure rise-for example, with a perfusion pressure between 10 and $30 \mathrm{mmHg}$-might be a partial ischaemia, enough to block transport but not enough to kill the axons. Or at pressure levels that are marginally damaging there may be alternating periods of blockade and recovery for individual axons, with shifting zones of ischaemia, with few or none of the axons reaching an irreversible stage of damage equivalent to 4 hours of total ischaemia.

This work relied heavily on the expert help of E. Barry Davis in conducting the experiments.

The investigation was supported in part by research grant EY-00031 and by National Research Service award EY -07021 , both awarded by the National Eye Institute. National Institutes of Health. Bethesda. Maryland. Dr Anderson is also supported by Research to Prevent Blindness, Inc., as a William and Mary Greve RPB International Research Scholar. Additional support was received from the National Glaucoma Research Program of the American Health Assistance Foundation.

\section{References}

1 Quigley HA. Anderson DR. The dynamics and location of axonal transport blockade by acute intraocular pressure elevation in primate optic nerve. Invest Ophthalmol Visual Sci 1976; 15: 606-16.

2 Anderson DR. Hendrickson A. The effect of intraocular pressure on rapid axonal transport in monkey optic nerve. Invest Ophthalmol Visual Sci 1974; 13: 771-83.

3 Minkler DS, Bunt AH, Johanson UW. Orthograde and retrograde axoplasmic transport during acute ocular hypertension in the monkey. Invest Ophthalmol Visual Sci 1977; 16: 426-41.

4 Levy NS. The effects of elevated intraocular pressure on slow axonal flow. Invest Ophthalmol Visual Sci 1974; 13: 691-5.

5 Minkler DS, Bunt AH, Klock IB. Radioautographic and cyto- 
chemical ultrastructural studies of axoplasmic transport in the monkey optic nerve head. Invest Ophthalmol Visual Sci 1978; 17: 33-50.

6 Gasterland D. Tanishima T. Kuwabara T. Axoplasmic flow during chronic experimental glaucoma. I. Light and electron microscopic studies of the monkey optic nerve head during development of glaucomatous cupping. Invest Ophthalmol Visual Sci 1978; 17: 838-46.

7 Radius RL, Anderson DR. Breakdown of the normal optic nerve head blood-brain barrier following acute elevation of intraocular pressure in experimental animals. Invest Ophthalmol Visual Sci 1980; 19: 244-55.

8 Radius RL, Schwartz EL, Anderson DR. Failure of unilateral carotid artery ligation to affect pressure-induced interruption of rapid axonal transport in primate optic nerves. Invest Ophthalmol Visual Sci 1980; 19: 153-7.

9 Kroll AJ. Experimental central retinal artery occlusion. Arch Ophthalmol 1968; 79: 453-69.

10 Hamasaki DI, Kroll AJ. Experimental central retinal artery occlusion. Arch Ophthalmol 1968; 80: 243-8.

11 Leone J. Ochs S. Reversibility of fast axoplasmic transport following different durations of axonic block in vitro and in vivo. Program/Abstracts, Annual Meeting of Society for NeuroScience. 1973; 3: 147.

12 Radius RL, Maumenee AE. Visual field changes following acute elevation of intraocular pressure. Trans Am Acad Ophthalmol Otolaryngol 1977; 83: 61-8.

13 Quigley HA. Anderson DR. Distribution of axonal transport blockade by acute intraocular pressure elevation in the primate optic nerve. Invest Ophthalmol Visual Sci 1977; 16: 640-4.

14 Minkler DS, Bunt AH, Johanson UW. Orthograde and retrograde axoplasmic transport in the monkey. Invest Ophthalmol Visual Sci 1977; 16: 426-41.

15 Radius RL, Anderson DR. Rapid axoplasmic transport in primate optic nerve: distribution of pressure induced interruption. Arch Ophthalmol 1981; 99: 650-4.

16 McLeod D. Marshall J, Kohner EM, Bird AC. The role of axoplasmic transport in the pathogenesis of retinal cotton-wool spots. Br J Ophthalmol 1977; 61: 177-91.

17 Ernest JT, Potts AM. Pathophysiology of the distal portion of the optic nerve. I. Tissue-pressure relationships. Am J Ophthalmol 1968; 66: 373-80.

18 Geijer C, Bill A. Effects of raised intraocular pressure on retinal, pre-lamina, lamina and retro-laminar optic nerve blood flow in monkeys. Invest Ophthalmol Visual Sci 1979; 18: 1030-42.

19 Anderson DR, Davis EB. Sensitivities of ocular tissues to acute pressure induced ischemia. Arch Ophthalmol 1975; 93: 267-74.

20 Anderson DR. Ascending and descending optic atrophy produced experimentally in squirrel monkeys. Am J Ophthalmol 1973; 76: 693-711.

21 Quigley HA, Davis EB, Anderson DR. Descending optic nerve degeneration in primates. Invest Ophthalmol Visual Sci 1977; 16: 841-9.

22 Radius RL, Anderson DR. Retinal ganglion cell degeneration in experimental optic atrophy. Am J Ophthalmol 1978; 86: 673-9. 